\title{
EXPLICITAÇÃO E OMISSÃO DE CATEGORIAS CULTURAIS EM FLORES RARAS E BANALÍSSIMAS E RARE AND COMMONPLACE FLOWERS: UM ESTUDO BASEADO EM CORPUS DE PEQUENA DIMENSÃO ${ }^{1}$
}

\author{
Alinne Balduino Pires Fernandes ${ }^{2}$ \\ Maria Lúcia Barbosa de Vasconcellos ${ }^{3}$
}

RESUMO: No contexto das relações tradutórias entre o Brasil e os Estados Unidos, investigam-se os fenômenos de explicitação e omissão de categorias culturais em um corpus paralelo bilíngüe de pequena dimensão, constituído de Flores raras e banalissimas (texto de partida - TP) (OLIVEIRA, 1995) e Rare and commonplace flowers (texto

\footnotetext{
Esta pesquisa é parte dos Projetos de Pesquisa Integrada e Institucional, intitulados (i) "Corpora, Gênero e (Re) Textualização: Interfaces nos Estudos da Tradução" (CNPq 551577/2992-9, coordenado pela Prof. Dra. Adriana Silvina Pagano) e (ii) "Tradução e Lingüística de corpora: em busca de interfaces com vistas a processos de categorização cognitiva e contextualização pragmática" (PROCAD/CAPES 097/01-2, coordenado pelos professores Dr. Fábio Alves - UFMG e Dra. Maria Lúcia Vasconcellos - UFSC). Os referidos projetos consorciam os trabalhos desenvolvidos no âmbito do Núcleo de Tradução - NUT (UFSC) e do Núcleo de Estudos da Tradução - NET (UFMG). Os resultados obtidos são fruto do trabalho de Iniciação Científica (CNPq) elaborado de agosto de 2003 a agosto de 2004, sob a orientação da Prof. Dra. Maria Lúcia Vasconcellos. A pesquisa será concluída como Trabalho de Conclusão de Curso (TCC), no fim de 2005. Resultados parciais estão disponibilizados no artigo intitulado "A omissão e a explicitação em Rare and Commonplace Flowers", Fernandes \& Vasconcellos, Anais Eletrônicos (CD-Rom) do III CIATI - III Congresso Ibero Americano de Tradução e Interpretação, 2004.

2 PIBIC - CNPq / Letras Inglês - Bacharelado/UFSC.

3 DLLE/PGET/PGI - UFSC
} 
de chegada - TC) (tradução de Besner, 2002). Esse estudo descreve as ocorrências de tais fenômenos no corpus examinado, visando a responder às seguintes perguntas de pesquisa: (i) qual a freqüência de explicitação e/ou omissão de itens culturais em Rare and Commonplace Flowers?; e (ii) que categorias culturais mais sofrem explicitações ou omissões? Como ferramenta para auxílio em buscas e contagem de freqüência, utiliza-se o software Wordsmith (Scott, 1998). Os resultados apontam para a tendência de preservar categorias culturais no TC, muitas vezes com o recurso da explicitação, em função de motivações contextuais.

UNITERMOS: abordagens discursivas aos Estudos da Tradução; estudos baseados em corpora; categorias culturais; explicitação; omissão.

ABSTRACT: This piece of research investigates the phenomena explicitation and omission of culture-specific items within the context of translational relations between Brazil and the USA. Flores raras e banalíssimas (source text - ST) (Oliveira, 1995) and Rare and commonplace flowers (target text - TT) (translated by Besner, 2002) compose the small scale bilingual parallel corpus of the research. This study describes the occurrence of those phenomena, focusing on the following research questions: (i) how often are culture-specific items made explicit/omitted in the TT? (ii) which culture-specific items have been most explicitated/omitted? The electronic tool used for item count is the software Wordsmith (Scott, 1998). Results show that the TT tends to maintain the culture-specific items of the ST, through the frequent use of explicitation due to contextual factors.

KEYWORDS: discursive approaches to Translation Studies; corpus-based studies; cultural categories; explicitation; omission. 


\section{Introdução}

No contexto das relações tradutórias Brasil-Estados Unidos, investigam-se os fenômenos de 'explicitação' e 'omissão' de categorias culturais em um corpus paralelo bilíngüe de pequena dimensão, constituído de Flores Raras e Banalissimas (TP) (Oliveira, 1995) e Rare and Commonplace Flowers (TC) (tradução de Besner, 2002). Esse estudo descreve as ocorrências de tais fenômenos no corpus examinado, visando a responder às seguintes perguntas de pesquisa: (i) qual a freqüência de explicitação e / ou omissão de itens culturais em Rare and Commonplace Flowers?; e (ii) que categorias culturais mais sofrem explicitações ou omissões?

O objetivo do trabalho é observar como se dá a tradução de termos culturais, no par de textos em questão, no contexto do contato tradutório/cultural Brasil-Estados Unidos. Segundo o tradutor ${ }^{4}$, o público-alvo do livro traduzido seria constituído de leitores de língua inglesa interessados na vida e obra da poetisa americana Elizabeth Bishop no Brasil e não, necessariamente, de especialistas em literatura. Assim, partiu-se do pressuposto de que, no discurso de origem e no texto traduzido, estariam em jogo duas representações, construídas a partir de contextos diferentes e atendendo a diferentes pautas.

O que informa o método como este trabalho tem sido desenvolvido são observações de pesquisadoras como Olohan (2002) e Magalhães (2002), apontando para a necessidade de investigação do comportamento lingüístico de traduções. Para isso, segundo elas, são necessárias investigações em corpora, a fim de ratificar ou corroborar hipóteses e interpretações, partindo-se de constatações derivadas de descrições lingüísticas em tradução, independentemente do par lingüistico envolvido. Como exemplo, cita-se a explicitation hypothesis (hipótese da explicitação) de Blum-Kulka (1986): ao explicar o fenômeno da explicitação como inerente ao trabalho do tradutor, em função de uma aparente necessidade de oferecer informações explicativas, a teóri-

4 Informação obtida em entrevista com Neil Besner em 7 de março de 2003, XVII ENPULI, Florianópolis (SC). 
ca discute a questão do tamanho das traduções, que tende a ser longas em relação ao texto de partida, independentemente da língua, do gênero ou registro.

No contexto da investigação do comportamento lingüístico de traduções por meio da investigação de um corpus em formato eletrônico, é feito, inicialmente, um levantamento quantitativo de aspectos textuais mais gerais, mencionados na seção 3. Tal levantamento foi possível pelo uso da suíte de programas Wordsmith Tools (Scott, 1998), a qual facilitou também a busca automática de elementos culturais no corpus em questão.

Nas próximas seções e subseções, serão apresentadas uma rápida revisão dos conceitos mencionados até aqui e que serão explorados deste ponto em diante, a descrição da metodologia e a discussão dos dados coletados no panorama geral do livro (i.e., o corpus), discussão desenvolvida, mais especificamente, nos capítulos 1, 2, 3, 4 e 24. A seção conclusiva tecerá considerações parciais obtidas até o presente, visto que a pesquisa ainda está em andamento.

\subsection{Os conceitos que informam o estudo}

A revisão bibliográfica, elaborada com vistas a este artigo, focaliza as noções básicas da nossa pesquisa, a saber: explicitação, omissão, categorias culturais e corpus. Tais noções são detalhadas, separadamente, abaixo.

\subsubsection{Explicitação}

O termo foi utilizado pela primeira vez por Vinay e Dalbernet (1958/1995). Em linhas gerais, é uma modalidade de tradução, que consiste em tornar explícito no texto de chegada (TC) o que está implícito no texto de partida (TP), devido ao contexto ou à situação discursiva. Shuttleworth e Cowie (1997:55) explicam o uso dessa modalidade de tradução em termos de inclusão de frases explicativas adicionais para auxiliar o fluxo lógico do texto e aumentar a legibilidade do mesmo. Nessa linha, Blum-Kulka (1986:19), em seu estudo sobre coesão e coerência em tradução, chega a afirmar que tal fenômeno se constitui em uma estraté- 
gia universal, independentemente do par de línguas em contato, inerente ao processo de mediação por meio da linguagem verbal, ou seja, da tradução.

$\mathrm{Na}$ verdade, o termo recebeu diversas interpretações desde sua cunhagem. No contexto brasileiro, por exemplo, Aubert (1998:107) define essa modalidade de tradução se em conjunto com a implicitação, uma vez que explicitação e implicitação seriam "duas faces da mesma moeda". Para ele, tanto a implicitação quanto a explicitação (em seus variados formatos: paráfrase, nota, aposto parentético) estão vinculadas à familiaridade suposta do leitor de chegada com o universo referencial veiculado. No contexto internacional, o termo é definido em Encyclopedia of Translation Studies, editado por Baker (2001:83), com base em quatro classificações: (a) explicitação obrigatória - utilizada para tornar o TC gramaticalmente aceitável na Língua de Chegada (LC); (b) explicitação opcional ditada por diferenças de estilos lingüísticos, por exemplo, o uso de conectivos, mudanças de pontuação; (c) explicitação pragmática - utilizada para explicitar questões culturais implícitas no TP, que podem não significar nada para o público-alvo do TC, pois não compartilham o mesmo conhecimento geral; (d) explicitação inerente ao processo de tradução - processo natural de qualquer tradução, devido à constante necessidade de formular idéias no TC concebidas no TP.

Vinculando, como Aubert (ibid.), a modalidade explicitação/ implicitação ao leitor do texto traduzido, este trabalho também adota, de Baker (ibid.), as noções de "explicitação pragmática", em função das categorias culturais observadas, e de "explicitação inerente ao processo de tradução", em função dos levantamentos estatísticos realizados.

\subsubsection{Omissão}

Segundo Baker (1992), caso uma expressão ou item em particular não seja vital para o desenvolvimento do texto em determinado contexto, sua omissão não produz dano. A omissão seria, portanto, uma modalidade legítima caso o objetivo maior seja produzir um texto mais "macio", de leitura mais fácil, do que procurar transmitir um significado mais "preciso" (Baker, 
1992:41-42). Ainda assim, há inevitavelmente alguma perda de significado na tradução.

Aubert (1998) distingue a omissão da implicitação, uma vez que, ao contrário da omissão - em que a informação contida no segmento não pode ser recuperada - a implicitação permite recuperação da informação, ainda que em outro segmento textual. $\mathrm{O}$ autor faz considerações interessantes sobre os possiveis motivos da omissão, que podem variar desde censura até limitações físicas de espaço, incluindo também "irrelevância do segmento textual em questão para os fins do ato tradutório específico" (idem, ibidem, p. 105).

Já para Vazquéz-Ayora (1977), a omissão pode ser vista como modalidade obrigatória, de maneira que obedeça às preferências de comportamento da LC. Nesse caso, as omissões são realizadas por méritos gramaticais e estilísticos, para não se traduzir demasiadamente, produzindo uma redundância. Além disso, para Shuttleworth e Cowie (1997), a omissão evita a inclusão de informações desnecessárias ou repetidas em um texto [idem, ibidem, p. 41, tradução nossa]. Dessa forma, recomenda-se analisar o conteúdo atentamente de modo a não comprometer o TP (Vazquéz-Ayora, 1977:366).

Dadas as explicações acima, definimos que este trabalho não abrangerá levantamentos de dados de omissões obrigatórias, visto que nosso objetivo é apontar tão somente as ocorrências de omissão pertencentes às categorias culturais especificadas abaixo. As omissões de natureza gramatical, com isso, não serão levadas em conta aqui.

\section{1. 3 Categorias culturais}

Cultura é um conceito complexo, cuja discussão está fora do escopo deste artigo. É necessário, entretanto, partir de uma definição desse conceito, para que seja possível o estabelecimento de categorias culturais a serem investigadas. Embora reconhecendo as reduções existentes na taxonomia de itens culturais proposta por Newmark (1988) - focada na consideração de aspectos de realia; ou seja, os referentes e suas expressões nos códigos de partida e de chegada - neste estudo adotamos a definição de "cultura" dada por esse pesquisador: estilo de vida e

TradTerm, 14, 2008, p. 73-101 
manifestações que são peculiares a uma comunidade que usa uma lingua particular e seus meios de expressão (idem, ibidem, p. 94, nossa tradução). Em particular, entendemos cultura como o estilo de vida e as manifestações que são peculiares, especificamente, à comunidade de classe média do Rio de Janeiro, Brasil, na época de Carlos Lacerda e, a partir dessa compreensão, exploramos os referentes culturais evidenciados na obra de Carmen Oliveira, conforme expressos no código carioca do português brasileiro de então.

Para fins de construção de ferramentas de análise para a pesquisa, cumpre especificarmos o que se entende por categorias culturais neste trabalho. Baseamo-nos na noção de culturespecific item (CSI) ['item de especificidade cultural', nossa tradução], proposta por Aixelá (1996). Interessa-nos na proposta de Aixelá o fato de que uma categoria cultural não existe por si só; antes é o resultado de um conflito que emerge na situação tradutória, a partir da necessidade de se traduzir um determinado texto. Esse problema pode surgir tanto da não-existência do elemento referido na LC, como da diferença de valores entre os elementos, caso existam em ambas as linguas.

As categorias culturais (ou itens de especificidade cultural) seriam, portanto, aquelas às quais teriam de reagir, de alguma forma, consciente ou inconscientemente, conforme o contexto tradutório em que estão trabalhando:

Aqueles itens realizados textualmente, cuja função e conotações em um texto-fonte envolvem um problema de tradução em sua transferência para um texto-alvo, sempre que tal problema seja um produto da inexistência do referido item ou de seu diferente status intertextual no sistema cultural dos leitores do texto alvo (Aixelá, 1996:58, tradução nossa).

Como a citação acima sugere, sempre que a tradução causar problemas que derivam de distância e/ou 'buracos' culturais entre a $\mathrm{LP}$ e a $\mathrm{LC}$ e seus respectivos contextos, o tradutor estará diante de uma categoria cultural ou, como o autor prefere, diante de um "item de especificidade cultural", que demandará uma tomada-de-decisão por parte do tradutor. 
Num contexto diferente, Medeiros (2003), estabeleceu categorias culturais para a investigação do tratamento dado a esses itens de especificidade cultural na legendagem de filmes brasileiros para o contexto americano, em sua tese de doutorado, no âmbito do Programa de Pós-Graduação em Letras/Inglês - PGI, da Universidade Federal de Santa Catarina. A autora adotou a visão de legendagem como "prática cultural", para investigar as possiveis inferências dos legendadores no que diz respeito às expectativas da audiência americana, o que, ela acredita, pode explicar os procedimentos de tradução observados nas legendas. Para o que Aixelá (1996) denominou itens de especificidade cultural', Medeiros (2003) propõe 'termos culturalmente amarrados' (culture-bound terms), que ela define como referências a componentes da cultural local cuja significação deriva menos do conteúdo proposicional da expressão e mais da especificidade referencial que eles representam (idem, ibidem, p. 11, nossa tradução). Embora num diferente cenário e utilizando diferente terminologia, Medeiros se aproxima de Aixelá no que tange o reconhecimento da existência de entidades/instâncias culturais que podem oferecer um problema de tradução, em termos de graus de opacidade, em função da distância entre o texto de partida e o leitor do texto de chegada.

Assim, as categorias de análise trazidas aqui foram construídas a partir da combinação das propostas de Aixelá (1996), Newark (1988) e Medeiros (2003). Além disso, a seleção das categorias culturais aqui investigadas foi também obtida através do que uma leitura preliminar de Flores raras $e$ banalissimas e Rare and commonplace flowers revelou ser potencialmente problemático no encontro/confronto lingüísticocultural possibilitado pelas relações tradutórias entre o Brasil e os Estados Unidos. Nominalmente, as categorias culturais que informaram o presente estudo são as 12 (doze) listadas a seguir:

“Topônimos": nomes de cidades, vilas, ruas, países, regiões; "Antropônimos": nomes das personagens e referências atribuídas a elas, como qualitativos; "Moedas"; "Comidas e Bebidas"; "Meios de transporte"; "Habitação"; "Vestimenta e Acessórios"; "Fauna e Flora" inclusive animais de estimação; "Referências religiosas"; "Instituições locais"; "Organizações políticas": partidos e títulos políticos; e "Lazer/ 
Trabalho", incluindo-se festas típicas, música e referências literárias.

\subsubsection{Corpus}

Recentemente, o uso de corpora nos Estudos da Tradução se estabeleceu como base para abordagens empíricas, às quais este trabalho se afilia. No contexto nacional, foram publicados dois volumes temáticos explorando a pesquisa nos Estudos da Tradução com base em corpora (de pequena e grande dimensão), ambos organizados pela pesquisadora Stella Tagnin (USP). São eles: Cadernos de Tradução - Tradução e Corpora (2003) e TradTerm - Tradução e Corpus - Translation and Corpora (2004). Os dois volumes agrupam as contribuições que estabeleceram a área no Brasil, apresentando, no dizer da organizadora, "uma amostragem do que se tem feito com corpora na área da tradução, em especial, mas não apenas, no Brasil” (Tagnin, 2004:7). Essas publicações estão em consonância com uma tendência semelhante, no cenário internacional, conforme atesta a publicação de Introducing Corpora in Translation Studies (Olohan, 2004). De forma similar, apresenta um mapeamento de métodos baseados em corpus na pesquisa em Estudos da Tradução, ao longo do qual são definidos tipos, desenhos e aplicações de corpora, incluindo-se um elenco de ferramentas computacionais para extração e análise de dados.

Nesse contexto, Baker (1995) aponta para uma mudança na definição do termo corpus o qual, na era pré-eletrônica, poderia significar qualquer conjunto de textos escritos com vistas ou não ao estudo lingüístico. Já na era da informática, corpus passa a ser entendido, em linhas gerais, como: (i) qualquer conjunto de textos em formato eletrônico passivel de análise semi-automática ou automática; (ii) textos escritos e textos falados; (iii) textos provenientes de fontes variadas, compilados para fins de estudo lingüístico com ou sem propósito específico de aplicação. Neste trabalho, adotamos a definição de Baker e, na direção do que propõe a autora, entendemos que existem três tipos básicos de corpora: 
i. Corpora multilingües: consistindo de dois ou mais corpora monolíngües em linguas diferentes entre si;

ii. Corpora paralelos: consistindo de textos de partida (TP) em uma língua $X$ e suas respectivas traduções, ou textos de chegada (TC), para uma língua $Y$;

iii. Corpora comparáveis: consistindo de dois ou mais corporana mesma língua, sendo um deles composto de textos escritos originalmente na língua em questão e o(s) outro(s) de traduções para a essa mesma língua, desde que pertencendo a uma variedade lingüistica semelhante e tendo sido escritos/falados em um período temporal semelhante.

No âmbito deste trabalho, abordamos, especificamente, o corpus paralelo e de pequena dimensão, uma vez que nosso conjunto textual é composto apenas do TP (Flores Raras $e$ Banalissimas, Oliveira, 1995) e do TC (Rare and Commonplace Flowers, tradução de Besner, 2002). Os textos do corpus pertencem ao gênero literário, mesclando biografia e ficção; além disso, é um corpus de público-alvo restrito (Olohan, 2004), diferentemente de corpus composto por textos jornalísticos, cujo público-alvo é muito amplo e diversificado.

\section{Método}

Primeiramente, tomou-se como base a leitura das teorias que informam os principais aspectos lingüísticos a serem investigados, bem como a leitura de Flores Raras e Banalíssimas e de Rare and Commonplace Flowers. Em seguida, com base na revisão de literatura, foram selecionadas possiveis categorias culturais a serem analisadas, com o intuito de delimitar o objeto de estudo.

A medida posterior foi a compilação do corpus, empregando-se o escaneamento dos livros em questão. Devido às incompatibilidades de configuração entre o resultado do escaneamento e o formato "doc" (document), houve necessidade de corrigir os textos, eliminando, especialmente, os problemas de ortografia e pontuação. 
Durante a revisão dessa formatação do corpus, incluímos a adequação aos padrões recomendados pelo CORDIALL ${ }^{5}$. Primeiramente, fizemos um levantamento de dados com os livros completos com vistas a um panorama geral do comportamento dos textos. Entretanto, o processo atual consiste em análise mais detalhada. Para tanto, cada arquivo (ou capitulo dos livros) foi convertido em formato "txt" (text), requerido pelo software empregado na pesquisa.

O primeiro levantamento utilizando o software WordSmith Tools foi feito com os textos completos, quais sejam, TP e TC. Excetuaram-se partes como prefácio, nota do tradutor, sumário, apêndices e referências bibliográficas, uma vez que tais essas, aqui consideradas paratextuais, não constituem o objeto de nossa pesquisa. Os aspectos levados em consideração, inspirados em Munday (2002), foram: (a) tokens, seqüências de letras com um espaço ortográfico de cada lado, cuja contagem levounos ao número total de palavras do corpus; (b) types, número de formas de palavras. Por exemplo, em "andava perambulando de hotel em hotel em Nova York", há 9 tokens e 7 types ("andava"; "perambulando"; "de"; "hotel"; "em"; "Nova"; "York"); (c) type/ token ratio, possivel indicador da variação lexical dos textos; (d) sentences, número de períodos; (e) sentence length, média do comprimento dos períodos pelo número de tokens; (f) paragraphs, número de parágrafos; $(\mathrm{g})$ paragraph length, média do comprimento dos parágrafos pelo número de tokens. Adicionalmente, foram calculadas diferenças percentuais (DP) de cada item - [(TP $-100 \%) /(\mathrm{TC}-\mathrm{x})]$ - utilizando-se regra de três simples. Excetuando-se os cálculos de DP, todos os outros foram feitos pela funcionalidade Word Count (contagem de palavras) do Wordsmith Tools.

Posteriormente, realizamos o levantamento dos itens mencionados nos capítulos Boston 1978 (1), Ó, Turista (2), O Pendúnculo Indecente (3), Era uma vez um rei chinês (4) e Boston 1978 (24). Cada capítulo foi arquivado com as informações do

5 CORDIALL (Corpus Discursivo para Análises Lingüísticas e Literárias): banco de dados desenvolvido na FALE / UFMG, em parceria com a UFSC. 
levantamento estatístico. Como forma de priorizar as categorias culturais, neste trabalho optamos por agrupar as categorias com os pontos de entrada "modalidades" e "categorias culturais", o que gerou uma tabela de dados.

As informações dessas tabelas foram coletadas por meio do Alignment ('alinhamento') automático disponivel no software. $\mathrm{O}$ 'alinhamento' reorganiza os textos, de modo que um período do TP é seguido de um período do TC, e assim sucessivamente. No entanto, optou-se pelo alinhamento de parágrafos - em vez do alinhamento por períodos, que afasta o período do TC do TP na visualização -, uma vez que este permite capturar, na descrição, momentos em que o tradutor transforma trechos segmentados do TP em períodos compostos; assim, a leitura dos textos e a coleta semi-automática de dados foram facilitadas.

A partir do próprio programa WordSmith Tools, os alinhamentos foram salvos em formato "txt" para serem manipulados pelo editor de textos Bloco de Notas. Optou-se aqui por uma rotulação numérica (Scott, 1998), que permitiu ao WordSmith Tools agrupar as modalidades por meio da funcionalidade Concordancer, automatizando, portanto, a contagem desejada nesta pesquisa. As modalidades foram rotuladas da seguinte maneira: Explicitação [1], no TC; Omissão [2], no TP; Tradução para o Inglês [3], no TC; e Forma mantida [4], no TC.

O programa agrupa as modalidades pela função Search Word, do Concordancer e, como resposta, conta as ocorrências. Para obter os dados rotulados como "Explicitação", "Omissão" e "Tradução para o inglês", utilizamos o Search Word. A seguir, no texto alinhado, utilizou-se a função editar > localizar para cada item agrupado pelo programa. Dessa forma, a localização das traduções foi simplificada. Para organizar todas as "formas mantidas" em tabelas, bastou agrupá-las com o Concordancer e contar as ocorrências repetidas. Em seguida, as "formas mantidas" foram colocadas em suas respectivas tabelas. Caso houvesse algum item específico em mente, como por exemplo, os antropônimos de freqüência mais alta, "Bishop" e "Lota", a modalidade consistia em digitar em Search Word a "palavra ou expressão requerida + [4]". Assim, o Concordancer agrupava todas as ocorrências e contava o número de vezes em que o item requerido apareceu no texto. 
Para evitar um alongamento exaustivo e desnecessário, optou-se, neste artigo, pela transcrição de excertos textuais em vez de apresentação das tabelas construídas no decorrer da pesquisa. A soma das contagens das categorias culturais das modalidades relevantes (Explicitações, Omissões, Formas Mantidas e Traduções para o Inglês) serão expostas brevemente, sob a forma de gráfico, na subseção 3.2.

\section{Discussão dos dados}

Nesta seção, serão discutidos dados levantados nos capítu$\operatorname{los} 1$, 2, 3, 4 e 24 das obras em questão. Esses dados serão discutidos separadamente e, além disso, serão comparados às contagens do panorama geral dos livros, apresentadas inicialmente.

\subsection{Dados quantitativos dos capitulos 1, 2, 3, 4 e dos livros completos}

\subsubsection{Livros completos}

Com o auxílio da ferramenta WordSmith Tools, foram feitos levantamentos quantitativos (contagens) de tokens, types, type/ token ratio, sentences, sentence length, paragraphs e paragraph length nos livros completos e em cada capitulo separadamente. Com o intuito de comparar os resultados obtidos em cada caso, utiliza-se a tabela 1. A análise comparativa pode propiciar a verificação do comportamento da linguagem do livro traduzido.

Inicia-se a discussão com a apresentação da Tabela 1, que indica as contagens do panorama geral do TP e do TC:

\begin{tabular}{|l|l|l|r|}
\hline & TP & DP & \multicolumn{1}{l|}{ TC } \\
\hline Tokens & $62.939,00$ & $<9,05 \%<$ & $68.637,00$ \\
\hline Types & $11.287,00$ & $>23,14 \%>$ & $8.675,00$ \\
\hline Type/Token Ratio & 17,93 & $>29,50 \%>$ & 12,64 \\
\hline Sentences & $5.155,00$ & $>4,02 \%>$ & $4.948,00$ \\
\hline Paragraphs & 11,88 & $<12,79 \%<$ & 13,40 \\
\hline Paragraph Length & $1.651,00$ & $<0,48 \%<$ & $1.659,00$ \\
\hline
\end{tabular}

Tabela 1: Panorama geral - dados quantitativos dos livros completos 
Conforme explicado na seção 2, types indicam o número das diferentes formas de palavras nos textos. Para Munday (2002), o cálculo de type/token ratio pode ser um indício da variação lexical do corpus. Assim, de acordo com a tabela 1, o TP teria uma variação lexical 29,5\% mais elevada que o TC. Munday (2002) utilizou essa ferramenta para comparar o TP e o TC em termos de correspondências, o que pode levar à identificação de possiveis omissões.

As contagens feitas para a obtenção do número de períodos (sentences), do número de parágrafos (paragraphs) e das respectivas médias (sentence length e paragraph length) podem marcar o estilo da escritora. Portanto, as diferenças percentuais (DPs) (número de períodos: TP $>4,02 \%>\mathrm{TC}$ ) sinalizam uma mudança de estilo (1) ou uma adequação ao sistema de pontuação da língua inglesa (2). Essa hipótese é explorada por Munday (2002) em suas análises de traduções do espanhol para o inglês. No corpus em estudo nesta pesquisa, isso pode ser verificado nos exemplos abaixo:

(1) Mudança de estilo:

(TP) Só. Já que só estava.

(TC) Alone, since she was, in fact, alone.

\section{(Excerto 1)}

(2) Adequação ao sistema de pontuação da língua inglesa:

(TP) Inocente do que estava para acontecer com sua vida depois do que ia propor, Mary informou, afavelmente:

- O apartamento é seu, pelo tempo que se demorar no Rio.

(TC) With no sense of the eventual implications for her life of the gesture, Mary made a friendly offer: "The apartment is yours for as long as you'd like to stay in Rio."

(Excerto 2)

Por meio do alinhamento, pode-se diagnosticar o motivo de ocorrências como as verificadas nos excertos acima. Com base 
nos capítulos analisados até o momento, pode-se afirmar que o Excerto 1 é uma demonstração de comportamento constante do tradutor: frases curtas do TP são reunidas em um só período no TC. Em outras palavras, o que é fragmentado no TP, é agregado no TC, o que remete a uma escrita mais formal e mais densa, em oposição a uma escrita mais impactante do TP.

No caso do Excerto 2, o WordSmith conta o travessão (-) como um novo período. Assim, o fato de, no texto em inglês, por força da convenção para registro de diálogos, ter sido empregados os dois pontos (:) e aspas ("”), faz com que esse trecho tenha sido reconhecido como um só período, o que, juntamente com a mudança de estilo, comprova o número menor de períodos no TC.

As médias mais elevadas do número de palavras nos períodos e parágrafos do $\mathrm{TC}(\mathrm{DP}=8,53 \%)$ podem ser explicadas também por ocorrências de explicitação, como observado no Excerto 3, abaixo:

(TP) de frente para a Avenida Atlântica

(TC) looking out onto the beach side, Avenida Atlântica

(Excerto 3)

O número de parágrafos também é um pouco maior no TC que no TP $(0,48 \%)$, provavelmente por causa das adaptações ao sistema de pontuação da língua inglesa.

\subsubsection{Comparações dos dados quantitativos dos capitulos $1,2,3,4$ e 24}

Por meio da função Word Count, foi elaborada uma tabela de levantamentos estatísticos preliminares, seguindo os mesmos critérios da Tabela 1, em todos os capítulos dos livros analisados até o momento. Os dados obtidos podem ser verificados abaixo: 


\begin{tabular}{|c|c|c|c|}
\hline Capítulos 1 & TP & DP & TC \\
\hline Tokens & 344,00 & $>0,58 \%>$ & 342,00 \\
\hline Types & 214,00 & $>6,07 \%>$ & 201,00 \\
\hline Type/Token Ratio & 62,21 & $>5,53 \%>$ & 58,77 \\
\hline Sentences & 37,00 & $>16,22 \%>$ & 31,00 \\
\hline Sentence Length & 8,54 & $<12,53 \%<$ & 9,61 \\
\hline Paragraphs & 17,00 & $=$ & 17,00 \\
\hline Paragraph Length & 19,65 & $<2,39 \%<$ & 20,12 \\
\hline Capítulos 2 & $\mathbf{T P}$ & DP & $\mathrm{TC}$ \\
\hline Tokens & $2.883,00$ & $<6.35 \%<$ & $3.066,00$ \\
\hline Types & $1.241,00$ & $>10,96 \%>$ & $1.105,00$ \\
\hline Type/Token Ratio & 43,05 & $>16,28 \%>$ & 36,04 \\
\hline Sentences & 258,00 & $>6.59 \%>$ & 241,00 \\
\hline Sentence Length & 10,97 & $<10.67 \%<$ & 12,14 \\
\hline Paragraphs & 109,00 & $=$ & 109,00 \\
\hline Paragraph Length & 26,45 & $<6,35 \%<$ & 28,13 \\
\hline Capítulos 3 & $\mathbf{T P}$ & DP & $\mathrm{TC}$ \\
\hline Tokens & 3.468 & $<9,20 \%<$ & 3.737 \\
\hline Types & 1.436 & $>10,38 \%>$ & 1.287 \\
\hline Type/Token Ratio & 41,41 & $>16,83 \%>$ & 34,44 \\
\hline Sentences & 310 & $>5,48 \%>$ & 293 \\
\hline Sentence Length & 11,04 & $<14,22 \%<$ & 12,61 \\
\hline Paragraphs & 103,00 & $>0,97 \%>$ & 102,00 \\
\hline Paragraph Length & 33,67 & $<8,05 \%<$ & 36,38 \\
\hline Capítulos 4 & $\mathbf{T P}$ & DP & $\mathrm{TC}$ \\
\hline Tokens & $2.074,00$ & $<8,73 \%<$ & $2.225,00$ \\
\hline Types & 968,00 & $>7,33 \%>$ & 897,00 \\
\hline Type/Token Ratio & 46,67 & $>13,63 \%>$ & 40,31 \\
\hline Sentences & 144,00 & $>6,25 \%>$ & 135,00 \\
\hline Sentence Length & 13,37 & $<14,96 \%<$ & 15,37 \\
\hline Paragraphs & 47,00 & $>4,26 \%>$ & 45,00 \\
\hline Paragraph Length & 44,13 & $<12,03 \%<$ & 49,44 \\
\hline Capítulos 24 & $\mathbf{T P}$ & DP & $\mathbf{T C}$ \\
\hline Tokens & 214,00 & $<12.62 \%<$ & 241,00 \\
\hline Types & 139,00 & \begin{tabular}{|l|}
$<8.63 \%<$ \\
\end{tabular} & 151,00 \\
\hline Type/Token Ratio & 64,95 & $>3.53 \%>$ & 62,66 \\
\hline Sentences & 27,00 & $=$ & 27,00 \\
\hline Sentence Length & 7,85 & $<12.74 \%<$ & 8,85 \\
\hline Paragraphs & 8,00 & $=$ & 8,00 \\
\hline Paragraph Length & 26,75 & $<<12.64 \%<$ & 30,13 \\
\hline
\end{tabular}

Tabela 2: Dados quantitativos dos capítulos 1, 2, 3, 4 e 24 
Diferentemente do panorama geral do livro e dos capítulos 2, 3, 4 e 24, as contagens do número de palavras (tokens) dos capítulos 1, "Boston 1978" (TP) e "Boston 1978" (TC), mostram uma incidência um pouco mais acentuada no TP do que no TC. Entretanto, o cálculo para uma possivel especulação da variação lexical (type/token ratio) dos textos indica um percentual um pouco mais elevado para o TP em todos os capítulos analisados até o momento, assim como nos experimentos feitos com os livros completos. As palavras diferentes (types) têm incidência maior nos TP, exceto nos capítulos 24, "Boston 1978" e "Boston 1978".

No que se refere ao número de períodos (sentences), os TP dos capítulos relacionados acima (tabela 2) apresentam número mais elevado do que os TC, exceto os capitulos 24, os quais apresentam quantidades iguais de períodos. Assim como demonstrado na subseção 3.1.1, o número mais baixo de períodos no TC pode ser explicado por uma mudança de estilo. Em relação às médias dos períodos (sentence length), os capítulos analisados individualmente se comportam da mesma maneira que o panorama geral dos livros: a média do TC é mais alta que a do TP.

Quanto ao número de parágrafos (paragraphs), os TP dos capítulos 3 e 4, bem como o TP completo, têm número mais alto de parágrafos; já os TP dos capítulos 1, 2 e 24 apresentam igual quantidade em relação ao TC. A média dos parágrafos (paragraph length) de todos os capitulos, bem como o panorama geral, demonstra que o TC é mais acentuado que o TP. Essa média mais acentuada dos TCs pode ser uma demonstração tanto da necessidade de explicar o TP para o público-alvo, o que remete à idéia de explicitação inerente ao processo de tradução (conforme mencionado em 1.1.1), quanto do estilo do tradutor, como comentado em 3.1.1, um tradutor que prefere reunir trechos fragmentados.

\subsection{Comparação geral dos dados obtidos nos capitulos 1,2 , 3,4 e 24}

Com vistas a proporcionar uma visão panorâmica dos dados coletados até o momento, elaboramos o gráfico a seguir. Os valores correspondentes para cada modalidade (Explicitação, 
Omissão, Tradução para o inglês e Forma mantida) foram obtidos pela soma das ocorrências desses fenômenos em cada capítulo. Tais valores foram coletados a partir das tabelas mencionadas na seção 2 .

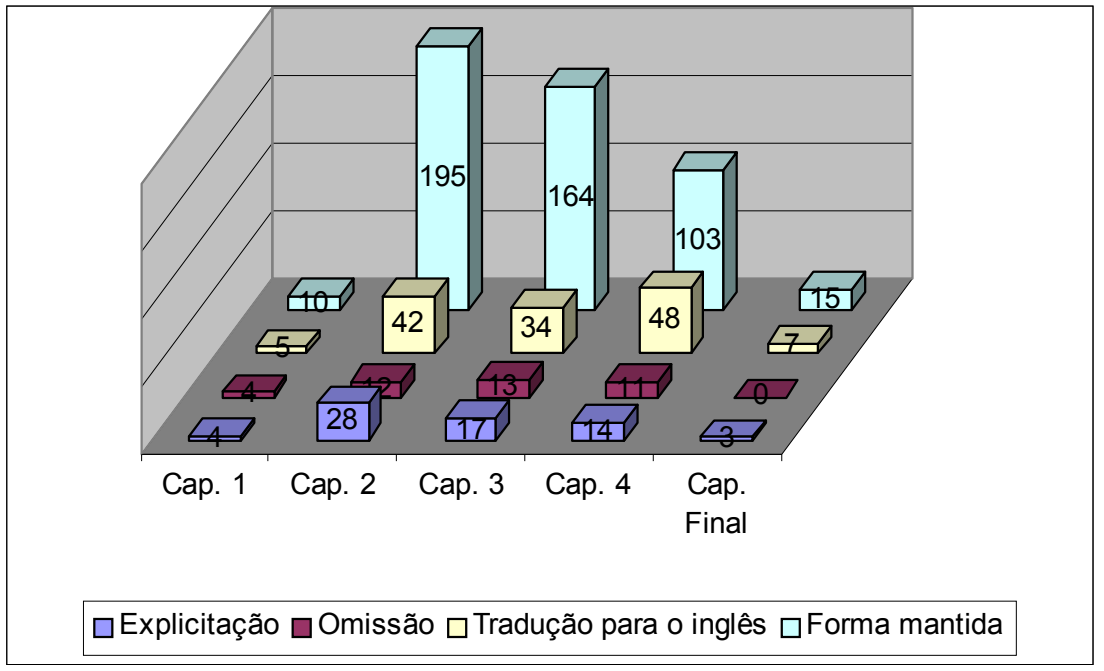

Gráfico 1: Visualização do panorama geral das modalidades nos capitulos $1,2,3,4$ e 24

Entendemos que o gráfico acima é uma demonstração da tendência de comportamento da linguagem no TC. Verifica-se, pois, um valor relativamente mais elevado para Forma Mantida em relação às demais modalidades. Isso se deve ao fato de todo nome mantido ser considerado como forma mantida, seja esse estrangeiro ou não para o TC, como, por exemplo, o nome da protagonista americana, "Bishop".

Note-se que os valores obtidos nos capítulos 1 e 24 são muito inferiores em relação aos demais, por serem capítulos com uma página apenas. Portanto, o tamanho do capítulo influencia diretamente no número de ocorrências desse tipo de fenômeno.

Cumpre mencionar, por oportuno, que esse gráfico foi inspirado pela proposta de Munday (2002), a qual sugere que os dados quantitativos podem ser uma sugestão de comportamen- 
to do TC em relação ao TP. Entretanto, não se deve julgar o TC sem as devidas contextualizações de seus valores.

A título de exemplificar os resultados verificados no Gráfico 1 , excertos textuais que foram organizados por esse procedimento serão transcritos abaixo.

\subsubsection{Ocorrências de Explicitação}

Nesta subseção, apresentam-se alguns exemplos de explicitação retirados dos capítulos 1, 2, 3, 4 e 24 com o auxílio da ferramenta Alignment. A seguir, no Excerto 4, apresentamos um exemplo de explicitação de Topônimo, retirado do capítulo 1:

(TP) Tinha detestado o emprego na Biblioteca do Congresso, em Washington.

(TC) She'd hated her job at the Library of Congress in Washington, D.C.

(Excerto 4)

Para evitar que o público-alvo enfrente problemas de compreensão, no TC, "Washington" é a capital dos EUA, explicitado por "D.C.”, e não o estado de Washington.

Prosseguindo-se para o capítulo 2, demonstra-se um exemplo de explicitação de Antropônimo:

(TP) Não deixou transparecer que, vulnerável como estava, por dentro marejava.

(TC) She didn't want Morse to sense how at sea she felt.

(Excerto 5)

É interessante notar que a afirmação em português não permite ao leitor constatar para quem Bishop não deixava "transparecer" como se sentia. Já o TC torna evidente o objeto em questão.

Abaixo segue mais um exemplo de explicitação de Antropônimo:

(TP) Era uma marchinha do carnaval de 22, proibida por fazer alusão desairosa ao candidato Arthur Bernardes. 
(TC) It was a little Carnival tune from 1922, censored because of its uncouth allusions to the frequent drinking bouts of the candidate Arthur Bernardes.

\section{(Excerto 6)}

Considera-se a ocorrência acima um antropônimo por ser comentário adicional por parte do tradutor a respeito do antropônimo "Arthur Bernardes". Aqui a explicitação contextualiza o leitor do TC, explicando a motivação da "marchinha", que satirizava o candidato.

Logo adiante, no capitulo 4, ressalta-se um exemplo de explicitação de "Comidas /Bebidas":

(TP) Antes de pegar o trem de volta, Arnaldo invariavelmente passava pela confeitaria Irmãos D'Angelo, para comprar caramelos para a filha.

(TC) Before catching his train back, Arnaldo invariably went by the D'Angelo Brothers bakery, to buy caramels, a Petrópolis specialty, for his daughter.

(Excerto 7)

Por meio de uma entrevista com o tradutor, obteve-se a informação de que este viveu no estado do Rio de Janeiro por alguns anos. Talvez em função disso, a qualificação dos caramelos como "a Petrópolis specialty" seja opinião pessoal do tradutor, o que não deixa de ser uma instância de explicitação da mensagem.

Para finalizar esta subseção, apresentamos um exemplo da categoria "Lazer/Trabalho", extraída do capítulo 24:

(TP) Gostava de Caetano Veloso, de "Não identificado".

(TC) She liked Caetano Veloso; she loved his song "Unidentified".

(Excerto 8)

Talvez partindo do pressuposto de que o público-alvo não fosse familiarizado às músicas de Caetano Veloso, além de traduzir o título, o tradutor apresentou a explicação: "his song". 


\subsubsection{Ocorrências de Omissão}

A modalidade de omissão teve um percentual relativamente baixo de ocorrência frente às demais. Nesta subseção, mostram-se exemplos de omissão retirados de cada capítulo.

Das quatro omissões do capítulo 1, Boston 1978, exemplifica-se uma omissão de topônimo:

(TP) Olha pela janela. Bela vista do porto de Boston.

(TC) She looks out the window at a beautiful view of the port.

(Excerto 9)

O excerto 9, acima, mostra um exemplo de omissão curioso: o de um item familiar à cultura de chegada. O dado acima não interfere no foco desta pesquisa, que é o de identificar como se dá a representação cultural do Brasil para o público-alvo da tradução. Entretanto, o exemplo acima pode ser lido como uma forma de demonstrar que não houve uma valorização suficiente da cultura local do TC.

Prosseguindo-se para o capítulo 2, encontramos um exemplo de omissão de um poema, pertencente à categoria "Lazer/ Trabalho":

(TP) Bishop correu as páginas, escolheu "Casamento".

- Eva: linda mulher - quando a conheci era tão formosa que me sobressaltei, capaz de escrever simultaneamente em três línguas - inglês, alemão e francês - e conversar ao mesmo tempo; igualmente categórica ao exigir animação e ao estipular silêncio: "Eu gostaria de ficar sozinha"; ao que retruca o visitante: "Eu gostaria de ficar sozinho; por que não ficamos sozinhos juntos?"

A cada estrofe Bishop consultava Lota com os olhos claros.

(TC) Bishop leafed through the pages and read several sections from "Marriage". At each stanza, Bishop lifted her light eyes to look at Lota.

(Excerto 10)

O trecho do poema omitido acima é uma tradução do poema "Marriage", de Marianne Moore. Ao invés de simplesmente 
inserir o poema original no TC, o tradutor optou por excluí-lo. Possivelmente, a omissão ocorreu pelo fato de o tradutor acreditar na familiaridade do público do TC com poema.

Já do capítulo 3 extraímos um excerto que diz respeito a Antropônimos:

(TP) - Como foi que seus pais morreram?

Bishop sentiu a velha pontada. O carinho de Lota a encorajava, porém.

- Meu pai morreu quando eu tinha oito meses.

(TC) "How did your parents die?"

"My father died when I was eight months old."

(Excerto 11)

O excerto diz respeito às sensações de uma das protagonistas, Bishop, a renomada poetisa americana, e também ao modo como a brasileira Lota, outra protagonista de central importância narrativa, se relacionava com aquela.

Do capítulo 4, exemplificam-se omissões de Topônimos (em negrito):

(TP) Gostava de ver as mansões belíssimas e conhecia a casa de Joaquim Rolas na Rua Ipiranga, a casa de Santos Dumont na Praça Rui Barbosa, a casa onde Stefan Zweig e a mulher se suicidaram, na Rua Gonçalves Dias, e, é claro, a Residência Imperial, que abrigava os descendentes da Coroa brasileira.

(TC) He liked to see the beautiful mansions, and he knew the house of Santos Dumont, honored by Brazilians as the inventor of the airplane, and the house in which Stefan Zweig and his wife committed suicide. He liked the Imperial Residence, which sheltered the descendants of the Brazilian monarchy.

(Excerto 12)

É interessante notar, no exemplo acima, que nomes não considerados como conhecidos internacionalmente foram omitidos no TC. Ao descrever as ruas da cidade, a autora menciona 
nomes de personagens da história brasileira, como "Joaquim Rolas", "Rui Barbosa", os quais, provavelmente, o tradutor não julgou necessário manter no TC, para não quebrar a fluência do texto traduzido. Entretanto, "Santos Dummont", nome provavelmente mais conhecido no âmbito internacional, foi até mesmo explicitado como se pôde notar no trecho sublinhado.

Quanto ao capítulo 24 de Rare and Commonplace Flowers, não houve ocorrências de omissão.

\subsubsection{Ocorrências de Tradução para o Inglês}

O que justifica a decisão de verificar ocorrências de itens traduzidos para o inglês é a possibilidade de se contrastar ocorrências em que o tradutor optou por manter os itens na forma original (Forma Mantida) com itens que tenham merecido alguma explicação (Explicitação), ou com os que tenham sido generalizados ou omitidos (Omissão).

No capítulo 1, encontramos a seguinte ocorrência:

(TP) Tinha detestado o emprego na Biblioteca do Congresso, em Washington.

(TC) She'd hated her job at the Library of Congress in Washington, D.C.

(Excerto 13)

O exemplo pode não parecer significativo, mas optou-se por transcrevê-lo para sinalizar a rotulação dos itens traduzidos de volta à língua original como um caso de Tradução para o Inglês. Por isso, os números exibidos no gráfico do item 3.2 geram uma visão superficial do comportamento do texto. Para uma visualização mais específica, deveriam ser levados em consideração somente itens próprios da cultura do TP, o que não ocorreu efetivamente.

Já no capítulo 2, verificam-se ocorrências classificadas como "Organizações Políticas" dentro dessa modalidade:

(TP) Lota contou que o imperador tinha escolhido aquela cidade para construir a residência de férias da familia real. 
(TC) Lota explained that the emperor had chosen this town to build the holiday residence of the royal family.

(Excerto 14)

Mais uma vez, ressalta-se que a escolha do excerto acima questiona o referencial da tradução para o público do TC em relação à imagem e história do Brasil. Mais um exemplo desse caso é apontado abaixo, extraído do capítulo 3:

(TP) Ao redor das senhorias, mestiços mal-vestidos falariam uma lingua incompreensivel.

(TC) All around their ladyships, scantily dressed mestizos spoke an incomprehensible language.

(Excerto 15)

O termo "mestiço" surge como resultado da miscigenação no Brasil, e é uma noção comum para o público leitor brasileiro. O que se argumenta aqui é se o público leitor em língua inglesa compreende "mestizo" como tal.

Do capítulo 4, segue abaixo uma ocorrência de "Topônimo":

(TP) O trem atravessava os subúrbios da Leopoldina e parava na Raiz da Serra.

(TC) It crossed the suburbs of Leopoldina and stopped at the foot of the mountain.

(Excerto 16)

A tradução literal de "Raiz da Serra" negligencia o fato de o termo ser, em português, o nome da região geográfica. Para o público-alvo do TC "the foot of the mountain" não passa de uma descrição do local.

No capítulo 24, há somente registros com o perfil do Excerto 14; ou seja, traduções de volta ao nome original em inglês, exemplos de nomes próprios da LC, ou de nomes locais que comumente recebem traduções, como, por exemplo, "Brasil" e "Brazil". 


\subsubsection{Ocorrências de Forma Mantida}

Em contraste com traduções cunhadas como "Brasil" e "Brazil", há exemplos de Topônimos que não possuem outra forma de expressão a não ser a original. Essa peculiaridade seria um caso de demonstração de cor local? Seguem os exemplos abaixo, retirados dos capítulos 1 e 24, respectivamente:

(TP) A primeira parada do navio foi em Santos.

(TC) The ship's first stop was at Santos.

(Excerto 17)

(TP) Nunca mais tinha voltado à casa de Petrópolis. Não ia voltar mais. Queria vender a casa de Ouro Preto.

(TC) She'd never gone back to the house in Petrópolis. She wouldn't ever go back again. She wanted to sell the house in Ouro Preto.

(Excerto 18)

O capítulo 2 também apresenta um exemplo que serve para ilustrar a presença da cor local:

(TP) Lota garantia que não, que a língua era dulcíssima e que com alguns estudos de botânica em pouco tempo Bishop estaria uma perita em rimas proparoxitonas.

- Quer ver? Lígula. Pétala. Plúmula - demonstrava ela, devagarzinho, marota.

(TC) Lota assured her it wasn't, that the language was very sweet and that with a little bit of botanical study, Bishop would soon become an expert in internal rhymes.

"Do you want to see? Ligula. Pétala. Plúmula," she pronounced, very slowly, roguishly.

(Excerto 19)

O excerto 19 acima procura ressaltar o som da língua do TC. Palavras usadas para ensinar Bishop tocam diretamente no leitor, que absorve um pouco da ressonância do português.

Adiante, com base em dados coletados no capítulo 3, insere-se um exemplo da modalidade para a categoria de "Topônimo" inconsistente: 
(TP) Lota contou que o pai havia comprado a Fazenda Samambaia quando ela tinha uns oito anos.

(TC) Lota said that her father had bought Fazenda Samambaia when she was about eight.

(Excerto 20)

Diz-se 'inconsistente' porque, em outras ocasiões, o tradutor opta por traduzir o tipo de edificação, seja "palácio" por "palace", ou "praças" por "squares" mas, no caso em questão, além de manter o nome específico do local, "Samambaia", mantém-se também "Fazenda". Talvez se justifique essa ocorrência pelo fato de se tratar de um lugar muito peculiar da narrativa, onde a maior parte dos eventos se desenrola.

Do capítulo 4, segue abaixo um exemplo de Forma Mantida em que o tipo de edificação fora traduzido, conforme mencionado no parágrafo acima, mas não o nome específico:

(TP) Na Estação Barão de Mauá, entre a Praça da Bandeira e São Cristóvão, Arnaldo de Oliveira aguardava o apito do trem para Ponte Nova, Minas Gerais.

(TC) At Baron of Mauá Station, between the squares of Bandeira and São Cristovão, Arnaldo de Oliveira waited for the whistle of the train.

(Excerto 21)

Há também uma ocorrência de omissão no excerto acima ("para Ponte nova, Minas Gerais"), mas não o abordaremos, já que o enfoque, aqui, se limita aos nomes mantidos na forma original.

\section{Reflexões finais}

Como mencionado na Introdução, os dados apresentados ao longo desta exposição são parte de um trabalho ainda em andamento, que será finalizado sob a forma de TCC.

$\mathrm{O}$ que se pode dizer, até o presente momento, é que se observou uma preocupação em explicar, e até conservar, alguns aspectos da cor local no TC, haja vista explicações acerca de 
"Santos Dummont" (Excerto 12) e dos "caramelos" (Excerto 7). Entretanto, não se pode afirmar que haja um comportamento constante no TC no tratamento das categorias culturais, como no caso de nomes omitidos (Excerto 12). Não se pode estabelecer critérios para as instâncias em que nomes são omitidos ou mantidos. Por exemplo, "praça Rui Barbosa" foi omitido, enquanto "Santos Dumont" foi mantido; ambos, porém, referem-se a "Topônimos". Pode-se apenas especular sobre as causas desse comportamento, como o fizemos acerca do Excerto 12. No caso de "Lazer/Trabalho", o mesmo tipo de inconsistência pode ser percebida quando um poema inteiro é omitido (Excerto 10), ao mesmo tempo em que uma cantiga é mantida (capítulo 3, vide Anexo 1), ainda que, no capítulo 4, possamos encontrar uma outra cantiga que é inteiramente traduzida para o inglês (vide Anexo 2).

Considerando-se os aspectos técnicos, o software WordSmith Tools é uma ferramenta que permite aos lingüistas perceber com muito mais facilidade determinados comportamentos lingüísticos. No caso do alinhamento, a mudança de estilo ficou muito clara. A ferramenta de contagem de palavras otimiza o tempo do pesquisador. Os números são fornecidos automaticamente, bastando apenas organizá-los, seja em uma tabela ou num gráfico, para manipulá-los da forma desejada.

Com isso, os dados das tabelas 1 e 2, por exemplo, comprovaram que o TC possui traços nítidos da hipótese da explicitação, devido a uma necessidade de explicar que é inerente ao processo de tradução. Por sua vez, os resultados obtidos com a ferramenta apontam para a tendência prevalecente de se preservar categorias culturais no TC, muitas vezes com o recurso da explicitação. A Tabela 2 sugere essa tendência e, de fato, tem sido comprovada até agora. A ocorrência de omissões é também significativa e, curiosamente, aponta para uma tendência a omitir os próprios elementos da cultura de chegada. Isso poderia ser justificado pelo que discute Aubert (1998), no que diz respeito à "irrelevância". Assim, os dados parciais examinados sugerem uma maior relevância conferida à aproximação do público-alvo à cultura de partida do que apresentar uma cultura já familiar a ele. 


\section{Referências Bibliográficas}

AIXELÁ, Javier Franco (1996) Culture-specific items in Translation. In: AVARRÉZ, Román \& VIDAL, M. Carmen-África (eds.). Translation, power and subversion. 1. ed. Clevedon: Multilingual Matters, p. 5278.

AUBERT, Francis (1998) Modalidades de tradução: teoria e resultados. In: TAGNIN, S.E.O. (1998), p. 99-128.

BAKER, Mona (1992) In other words. A coursebook on translation. 1. ed. London and New York: Routledge, p. 41-43.

(1995) Corpora in translation studies: an overview and some suggestions for future research. Target. Amsterdam, v. 7.2, p. 223243.

(2001) (ed.) Encyclopedia of Translation Studies. 1. ed. Manchester: Routledge.

BLUM-KULKA, Shoshana (1986) Shifts of cohesion and coherence in Translation. In: HOUSE, J. \& BLUM-KULKA, S. (ed.) Interlingual and intercultural communication. 1. ed. Alemanha: Gunter Naar Verlag Tubinger, p. 17-35.

MAGAlHÃES, Célia Maria \& BATISTA, Maria da Conceição (2001) Features in translated Brazilian-Portuguese texts: a corpus-based research. Cadernos da Tradução, v. 5, n. 9. Florianópolis: UFSC, p. 81-109.

MEDEIROS, Ritalice Ribeiro de (2003) Subtitling as culture planning and representations of foreign lands: Rotten suburbs, bucks and raw sugar in the English subtitles of Terra Estrangeira, Central do Brasil and Abril Despedaçado. Tese de Doutorado em Letras - Lingua Inglesa e Lingüística Aplicada. Florianópolis: UFSC, p. 147-167.

MUNDAY, Jeremy (2002) Systems in Translation: a systemic model for Descriptive Translation Studies. In: HERMANS, Theo (ed.), Crosscultural transgressions: research models in Translation Studies II: historical and ideological issues. Manchester: St. Jerome Publishing, p. 79-92.

NEWMARK, Peter (1988) A textbook of translation. 1. ed. New York: Prentice Hall International, p. 94-102.

OLIVEIRA, Carmem (1995) Flores raras e banalissimas. 1. ed. Rio de Janeiro: Rocco, 1995.

(2002) Rare and commonplace flowers. New Jersey: Rutgers University Press. Besner, Neil (trad.). 1. ed.

TradTerm, 14, 2008, p. 73-101 
OLOHAN, Maeve (2001) Leave it out! Using a comparable corpus to investigate aspects of explicitation in Translation. Cadernos da Tradução, v. 5, n. 9. Florianópolis: UFSC. p. 153-169. (2004) Introducing corpora in Translation Studies. New York: Routledge.

SCOTT, M. (1998) WordSmith Tools: Software Language Tools for Windows. Oxford: Oxford University Press.

SHUTTLEWORTH, M. et al. (1997) Dictionary of Translation Studies. 1. ed. Manchester: St. Jerome Publishing, p. 55-141.

TAGNIN, S.E.O. (2003) (ed.) Cadernos de Tradução. (2004) (ed.) TradTerm. São Paulo: CITRAT/FFLCH-USP.

VAZQUÉZ-AYORA, Gerardo (1977) Introducción a la Traductologia. Curso básico de Traducción. 1. ed. Georgetown: Georgetown University, p. 358-366.

VENUTI, Lawrence (1995) The translator's invisibility. 1. ed. London: Routledge.

VINAY, Jean-Paul \& DALBERNET, Jean (1995) Comparative stylistics of French and English. A methodology for Translation. 1. ed. SAGER, Juan C. \& HAMEL, M.-J. (trads. e eds.). Amsterdam/Philadelphia: John Benjamins Publishing Company, p. 342.

\section{ANEXOS}

Anexo 1 - retirado do capítulo 3:

(TP) Cantou, com entonação patriótica:

\section{Ai seu Mé}

Ai seu Mé

Lá no Palácio das Águias

Olé

\section{Não hás de pôr o pé}

Era uma marchinha do carnaval de 22, proibida por fazer alusão desairosa ao candidato Arthur Bernardes.

(TC) She sang, with patriotic fervor,

\section{Ai seu Mé}

Ai seu Mé

Lá no Palácio das Águias

Olé

Não has de pôr o pé. 